\title{
Human recombinant lactoferrin acts synergistically with antimicrobials commonly used in neonatal practice against coagulase-negative staphylococci and Candida albicans causing neonatal sepsis
}

\author{
Mohan Pammi Venkatesh ${ }^{1}$ and Liang Rong ${ }^{2}$ \\ ${ }^{1}$ Baylor College of Medicine \& Texas Children's Hospital, 6621 Fannin, MC: WT 6-104, Houston, \\ TX 77030, USA \\ ${ }^{2}$ Baylor College of Medicine, 1102 Bates Avenue, MC: BCM 320, Houston, TX 77030, USA
}

Correspondence

Mohan Pammi Venkatesh

mohanv@bcm.edu

Received 12 February 2008

Accepted 24 April 2008

\begin{abstract}
Neonatal sepsis causes significant mortality and morbidity. Coagulase-negative staphylococci (CoNS) and Candida frequently cause neonatal sepsis at $>72 \mathrm{~h}$ of age. Lactoferrin, which is present in human milk, is a component of innate immunity and has broad-spectrum antimicrobial activity. The synergistic effects of lactoferrin with antibiotics against neonatal isolates have not been systematically evaluated. Here, eight clinical strains (seven neonatal) of CoNS and three strains (two neonatal) of Candida albicans were studied. $\mathrm{MIC}_{50}$ and $\mathrm{MIC}_{90}$ values of human recombinant lactoferrin (talactoferrin; TLF), vancomycin (VAN) and nafcillin (NAF) against CoNS, and of TLF, amphotericin B (AMB) and fluconazole (FLC) against C. albicans, were evaluated according to established guidelines. Antimicrobial combinations of TLF with NAF or VAN against CoNS, and TLF with AMB or FLC against C. albicans, were evaluated by a chequerboard method with serial twofold dilutions. Synergy was evaluated by the median effects principle, and combination indices and dose reduction indices were reported at 50,75 and $90 \%$ inhibitory effect at several drug-dose ratios. It was found that TLF acted synergistically with NAF and VAN against CoNS, and with AMB and FLC against C. albicans, at multiple dose effects and drugdose ratios with few exceptions. In synergistic combinations, drug reduction indices indicated a significant reduction in doses of antibiotics, which may be clinically relevant. Thus TLF acts synergistically with anti-staphylococcal and anti-Candida agents commonly used in neonatal practice and is a promising agent that needs to be evaluated in clinical studies.
\end{abstract}

\section{INTRODUCTION}

Sepsis is a major cause of death in the neonatal period, especially in preterm and very-low-birth-weight neonates (birth weight $<1500 \mathrm{~g}$ ) (Kaufman \& Fairchild, 2004; Lawn et al., 2006). Sepsis significantly increases morbidity, prolongs the need for ventilation and intravascular access, increases the length of hospital stay and increases the incidence of bronchopulmonary dysplasia, necrotizing enterocolitis and adverse neurodevelopmental outcomes (Adams-Chapman \& Stoll, 2006; Stoll et al., 2002, 2004). Coagulase-negative staphylococci (CoNS) and Candida albicans are among the most common organisms isolated from neonatal sepsis (Stoll et al., 2002). The global emergence of antibiotic resistance (Levy, 1998, 2001) and immature neonatal host defences dictate the need to use

Abbreviations: $\mathrm{AMB}$, amphotericin $\mathrm{B}$; $\mathrm{Cl}$, combination index; CoNS, coagulase-negative staphylococci; DRI, dose reduction index; FLC, fluconazole; NAF, nafcillin; TLF, human recombinant lactoferrin (talactoferrin alpha); VAN, vancomycin. non-antibiotic agents that can enhance host immunity and can be used against neonatal sepsis. Lactoferrin, a component of innate immunity, is one such agent.

Lactoferrin is an $80 \mathrm{kDa}$, monomeric, diferric, cationic glycoprotein with an isoelectric point of between 8.4 and 9.0 (Moguilevsky et al., 1985; Sanchez et al., 1992) and belongs to the transferrin family of iron-binding glycoproteins. Lactoferrin is composed of 690 aa with a highly conserved three-dimensional structure, and its role in several pathophysiological functions including iron homeostasis, organ morphogenesis, host defence against infection, inflammation and cancer is emerging (Baker et al., 1998; Ward et al., 2005).

Lactoferrin is found on mucosal surfaces, in significant concentrations in human colostrum $\left(7 \mathrm{~g} \mathrm{l}^{-1}\right)$ and less so in mature human milk $\left(1 \mathrm{~g} \mathrm{l}^{-1}\right)$, tears $\left(3.8 \mathrm{~g} \mathrm{l}^{-1}\right)$, saliva $\left(20 \mathrm{mg} \mathrm{l}^{-1}\right.$ ), seminal fluid and secondary granules of neutrophils (Hennart et al., 1991; Masson et al., 1966, 1969; Masson \& Heremans, 1971). Normal serum levels in 
humans range from 0.4 to $2 \mathrm{mg}^{-1}$ and may increase to $200 \mathrm{mg} \mathrm{l}^{-1}$ following its release from neutrophils during sepsis (Bennett \& Kokocinski, 1978). The expression and secretion of lactoferrin in significant concentrations on mucosal surfaces and its release at inflammatory sites by polymorphonuclear neutrophils have established its role as an innate immunity agent, as well as a contributor to acquired cellular and humoral immune responses.

Lactoferrin has broad-spectrum antimicrobial activity against bacteria, fungi, viruses and protozoa. Its antimicrobial activity stems from two distinct effects: (i) its ironsequestering ability, which can be negated by saturation with iron (Kalmar \& Arnold, 1988; Yamauchi et al., 1993); and (ii) its iron-independent killing due to a direct interaction with the microbial surface resulting in cell lysis (Farnaud \& Evans, 2003; Orsi, 2004; Valenti \& Antonini, 2005).

Bovine lactoferrin has been shown to inhibit planktonic (free-living) organisms, as well as biofilms of Staphylococcus epidermidis growing on soft contact lenses in vitro (Leitch \& Willcox, 1999b). Both bovine and human lactoferrins inhibit Candida isolates from the oral cavity of immunocompromised patients by their action on Candida cell membranes (Xu et al., 1999) and exhibit synergy with common antifungal agents (Kuipers et al., 1999). Synthetic lactoferrin-derived peptides have been shown to have a greater antifungal effect compared with native lactoferrin in vitro, and the first two arginines at the $\mathrm{N}$-terminus of human lactoferrin appear to be critical in the candidacidal activity (Lupetti et al., 2000).

Synergistic effects of lactoferrin with antimicrobial agents against S. epidermidis and Candida species have been reported (Leitch \& Willcox, 1999b; Lupetti et al., 2003), but the term synergy in drug combinations has been loosely applied without definite modelling or derivations, and has not been evaluated at multiple dose effects or drug-dose ratios (Odds, 2003). The utility of combining human recombinant lactoferrin (talactoferrin alpha; TLF) with the commonly used antimicrobials in neonatal practice to enhance antimicrobicidal effects has not yet been reported.

\section{METHODS}

Antimicrobials. TLF expressed in Aspergillus awamori (Ward et al., 1995) was provided by Agennix. TLF stock solution (100 $\mathrm{mg} \mathrm{ml}^{-1}$ ) was diluted to give serial twofold dilutions starting from a concentration of $8300 \mu \mathrm{g} \mathrm{ml}^{-1}$. Fluconazole (FLC) stock solution ( $2 \mathrm{mg} \mathrm{ml}{ }^{-1}$; Sigma-Aldrich) was diluted to give serial twofold dilutions starting from $64 \mu \mathrm{g} \mathrm{ml}^{-1}$. Amphotericin B (AMB) obtained from Streptomyces (Sigma-Aldrich), vancomycin (VAN; USB) and nafcillin (NAF; Sigma-Aldrich) were used in serial twofold dilutions starting from $4 \mu \mathrm{g} \mathrm{ml}^{-1}$.

Organisms. The organisms used were S. epidermidis ATCC 55133, seven clinical strains of CoNS (S. epidermidis $100 \mathrm{H}$ and 101S, CoNS 102R, 103A, 104O, 105D and 106F) and C. albicans strains ATCC 32354, 200C and 201M. All clinical strains (except for S. epidermidis ATCC 55133 and C. albicans ATCC 32354, which are human non-neonatal isolates) were isolated from the peripheral blood of neonates with sepsis at Texas Children's Hospital, Houston, TX, USA.

Growth media. Sabouraud dextrose agar plates and glucose/yeast extract/peptone broth (GYEP) were used for Candida subcultures, and RPMI 1640 without bicarbonate buffered with 0.165 M MOPS to a $\mathrm{pH}$ of 7 was used for antimicrobial susceptibility testing. For CoNS, trypticase soy agar with $5 \%$ sheep blood and trypticase soy broth (TSB) were used for subcultures and antimicrobial susceptibility testing, respectively.

Inoculum preparation. CoNS and C. albicans were plated overnight from cryogenic stocks stored at $-80{ }^{\circ} \mathrm{C}$. Five colonies were picked and inoculated into TSB or GYEP broth, respectively $(75 \mathrm{ml}$ in a $250 \mathrm{ml}$ flask), and incubated for up to $2 \mathrm{~h}$ in an incubator shaker at $37{ }^{\circ} \mathrm{C}$, resulting in growth-phase organisms. The suspension was centrifuged at $1932 \mathrm{~g}$ for $10 \mathrm{~min}$ three times and the sediment was suspended in normal saline to an $\mathrm{OD}_{630}$ of 0.6 (corresponding to $0.5 \times 10^{8}-1 \times 10^{8}$ c.f.u. $\mathrm{ml}^{-1}$ ) for CoNS and an $\mathrm{OD}_{490}$ of 0.6 (corresponding to $1 \times 10^{7}-5 \times 10^{7}$ c.f.u. $\mathrm{ml}^{-1}$ ) for C. albicans. Quantitative cultures of the final suspension confirmed the final concentrations.

\section{Antimicrobial susceptibility testing of CoNS and C. albicans}

MICs of TLF, NAF and VAN against CoNS. MIC 50 and MIC 90 values were determined using the broth microdilution method as recommended by the CLSI $(2003,2007)$ with minor modifications. Five microlitres of a $1: 10$-diluted inoculum was added to $100 \mu \mathrm{l}$ of the serial dilutions of the antimicrobial to be tested in a 96-well microtitre plate. The final concentration of CoNS in each well of the microtitre plate was $2.5 \times 10^{5}-5 \times 10^{5}$ c.f.u. $\mathrm{ml}^{-1}$. Microtitre plates were incubated for $24 \mathrm{~h}$ at $35^{\circ} \mathrm{C}$. Drug-free TSB with organisms (growth control) and organism-free drug controls (sterility control) were used for comparison. Microtitre plates were incubated at $35{ }^{\circ} \mathrm{C}$ for $24 \mathrm{~h}$, and $\mathrm{MIC}_{50}$ and $\mathrm{MIC}_{90}$ values were estimated by a colorimetric method using 2,3-bis(2-methoxy-4-nitro-5sulfophenyl)-5-[(phenyl-amino) carbonyl]-2H-tetrazolium hydroxide (XTT). Menadione [10 mM solution in acetone (99.9\% HPLCgrade) $]$ was added to XTT $\left(0.5 \mathrm{~g} \mathrm{l}^{-1}\right)$ to give a $1 \mu \mathrm{M}$ concentration of menadione and $100 \mu \mathrm{l}$ of this was added to the wells of 96-well microtitre plates and incubated in the dark for $2 \mathrm{~h}$. The plates were centrifuged for $5 \mathrm{~min}$ at $1932 \mathrm{~g}, 100 \mu \mathrm{l}$ supernatant was transferred to a new microtitre plate and the colour was read at $A_{490}$ in a microtitre plate reader (Cerca et al., 2005). End points at 50 and $90 \%$ inhibition of growth compared with the growth control were determined as $\mathrm{MIC}_{50}$ and $\mathrm{MIC}_{90}$ values, respectively. Experiments were carried out in duplicate on two different days and the means of the readings of XTT reduction were used to determine the $\mathrm{MIC}_{50}$ and $\mathrm{MIC}_{90}$.

MICs of TLF, AMB and FLC against C. albicans. $\mathrm{MIC}_{50}$ and $\mathrm{MIC}_{90}$ values were determined using the broth microdilution method as described by the CLSI (2002). C. albicans inoculum (100 $\mu \mathrm{l})$ was added to $100 \mu \mathrm{l}$ of serial twofold dilutions of the antifungal agent to be tested in 96-well microtitre plates. The final concentration of $C$. albicans was $0.25 \times 10^{3}-0.5 \times 10^{3}$ c.f.u. $\mathrm{ml}^{-1}$ in each well of the microtitre plate. Drug-free medium with organisms (growth control) and organism-free drug controls (sterility control) were used for comparison. Microtitre plates were incubated at $35{ }^{\circ} \mathrm{C}$ for $46 \mathrm{~h}$, and $\mathrm{MIC}_{50}$ and $\mathrm{MIC}_{90}$ values were estimated by a colorimetric method using XTT as described above. Experiments were carried out in duplicate on two different days and the means of the readings of XTT reduction were used to determine $\mathrm{MIC}_{50}$ and $\mathrm{MIC}_{90}$ values.

Antimicrobial susceptibility testing of CoNS and C. albicans using antimicrobial combinations with TLF. Antimicrobial combinations of TLF with VAN or NAF were tested against CoNS, and combinations 
of TLF with AMB or FLC against C. albicans. An $8 \times 8$ chequerboard matrix of the drugs $(50 \mu \mathrm{l}$ each) in serial twofold dilutions including zero concentrations on the $x$ - and the $y$-axes were set up in 96-well microtitre plates. As in the MIC determinations, $5 \mu \mathrm{l}$ CoNS suspension $\left(2.5 \times 10^{4}-7.5 \times 10^{4}\right.$ c.f.u. $\left.\mathrm{ml}^{-1}\right)$ or $100 \mu \mathrm{l}$ C. albicans suspension $\left(0.5 \times 10^{3}-1 \times 10^{3}\right.$ c.f.u. $\left.\mathrm{ml}^{-1}\right)$ was added to the wells containing the antimicrobial combinations. The plates were incubated at $35{ }^{\circ} \mathrm{C}$ for $24 \mathrm{~h}$ for CoNS and for $46 \mathrm{~h}$ for C. albicans. Inhibitory end points were determined by XTT reduction. Experiments were performed in duplicate on two different days and the means of readings of XTT reduction on each day were used to provide two data points for analyses.

Analyses of drug interactions in antimicrobial combinations. The inhibitory effects of the antimicrobials in combination with TLF were tabulated in constant ratios of each other including ratios of their $\mathrm{MIC}_{50}$ values (equipotency ratios). The median effects principle was used to study drug interactions and estimate combination indices (CIs) and dose reduction indices (DRIs) (Chou, 2006).

The median effect equation is given by $f_{\mathrm{a}} / f_{\mathrm{u}}=\left(D / D_{\mathrm{m}}\right)^{\mathrm{m}}$, where $f_{\mathrm{a}}$ is the fraction affected by the drug, $f_{\mathrm{u}}$ is the fraction unaffected $\left(f_{\mathrm{u}}=1-f_{\mathrm{a}}\right)$, $D$ is the concentration of the drug, $D_{\mathrm{m}}$ is the median-effect dose (the dose of the drug that produces $50 \%$ of the effect, $\mathrm{ED}_{50}$ ) and $m$ is the co-efficient signifying the shape of the dose-effect relationship, where $m=1, m>1$ and $m<1$ indicate hyperbolic, sigmoidal and flatsigmoidal dose-effect curves, respectively.

The CI value is given by the equation $\mathrm{CI}=D_{1} / D_{x 1}+D_{2} / D_{x 2}$, and the DRI for drug $1=D_{x 1} / D_{1}$ and for drug $2=D_{x 2} / D_{2}$, where $D_{1}$ and $D_{2}$ are doses of drug 1 and drug 2 in combination and $D_{x 1}$ and $D_{x 2}$ are doses of drug 1 and drug 2 that produce $x \%$ effect when used alone. A value of $\mathrm{CI}<1$ indicates synergy, $\mathrm{CI}>1$ indicates antagonism and $\mathrm{CI}=1$ indicates an additive effect. The DRI determines by how many fold a drug dose can be reduced in a synergistic drug combination for a given inhibitory effect on the organism. Multiple drug-dose effect calculations were performed using CalcuSyn software (Biosoft) using the constant ratios of the drug combinations.

\section{RESULTS AND DISCUSSION}

\section{Antimicrobial susceptibility of clinical strains of CoNS and C. albicans}

The $\mathrm{MIC}_{50}$ values of TLF against CoNS ranged from 500 to $2000 \mu \mathrm{g} \mathrm{ml}^{-1}$, but the $\mathrm{MIC}_{90}$ values were beyond the range tested for all strains except CoNS 106F (Table 1). For $C$. albicans, the $\mathrm{MIC}_{50}$ values of TLF ranged from 62.5 to $250 \mu \mathrm{g} \mathrm{ml}^{-1}$, but the $\mathrm{MIC}_{90}$ values were beyond the range tested for all three strains. The huge difference in concentrations between $\mathrm{MIC}_{50}$ and $\mathrm{MIC}_{90}$ values suggest a bacteriostatic effect rather than a bacteriocidal effect. Similar large differences were seen with the fungistatic FLC, where the $\mathrm{MIC}_{90}$ values were 1000 times greater than the $\mathrm{MIC}_{50}$ values. The differences between $\mathrm{MIC}_{50}$ and $\mathrm{MIC}_{90}$ for NAF (varying from the same concentration to 16-fold), vancomycin (same to 2-fold) and amphotericin (16-33fold) were not as pronounced as that of TLF or FLC.

Cationic lactoferrin probably acts by binding to anionic lipotechoic acid of S. epidermidis, thereby reducing its charge and allowing lysozyme to act (Leitch \& Willcox, 1999a). Lactoferrin at $1800 \mu \mathrm{g} \mathrm{ml} \mathrm{m}^{-1}$ together with lysozyme has been shown to be effective in vitro against S. epidermidis. In addition, lactoferrin alone at $200 \mu \mathrm{g} \mathrm{ml}^{-1}$ and in conjunction at $100 \mu \mathrm{g} \mathrm{ml}^{-1}$ with azole group antifungal agents inhibits C. albicans (Wakabayashi et al., 1996, 1998). In our experiments, we found that TLF alone inhibited $\left(\mathrm{MIC}_{50}\right)$ all strains of CoNS at concentrations of $500-2000 \mu \mathrm{g} \mathrm{ml}^{-1}$ and all strains of C. albicans at concentrations of $62.5-250 \mu \mathrm{g} \mathrm{ml}^{-1}$, which is similar to previously published studies (Lupetti et al., 2000, 2003).

Table 1. Antimicrobial susceptibility of clinical strains of CoNS and C. albicans

Antimicrobial susceptibility performed using the microdilution method in 96-well microtitre plates and an XTT reduction assay was used to determine inhibitory end points.

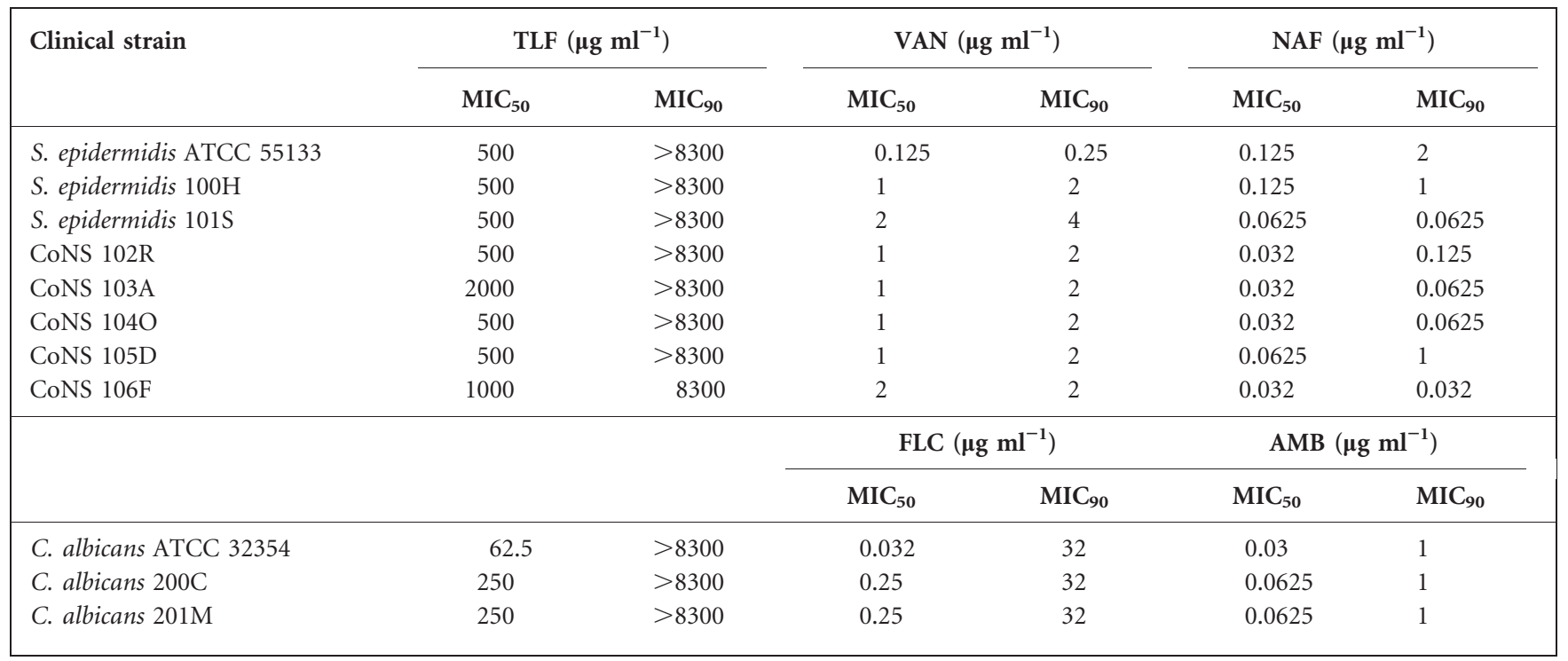


Lactoferrin may act in concert with other human defence mechanisms in vivo and may have an enhanced antimicrobial effect (Valenti \& Antonini, 2005). In a neonatal rat model of polymicrobial infection of $S$. epidermidis and C. albicans, TLF significantly improved survival (Venkatesh et al., 2007). Other investigators have reported the efficacy of TLF in animal models of systemic Escherichia coli infection (Edde et al., 2001) and of synthetic lactoferrinderived peptides in invasive Candida infections (Lupetti et al., 2007).

\section{Antimicrobial combinations with TLF}

There are many advantages of combining drugs in the treatment of infections, including enhancing efficacy, the ability to decrease the doses of antimicrobial agents (leading to a reduction in toxicity) and minimizing the development of resistance. The potential for the clinical use of lactoferrin in conjunction with antibiotics in the therapy or prevention of neonatal sepsis suggested that it would be useful to evaluate the drug interactions of TLF with antibiotics commonly used in neonatal practice.

Although many methods of evaluating synergy, an additive effect or antagonism in drug combinations exist, we used the median effects principle elucidated by Chou (2006), which is widely used in cancer and infection research (Berenbaum, 1989; Greco et al., 1995; Odds, 2003; Prichard et al., 1993). The advantage of this method is that it overcomes the assumption that drug interactions are linear across dosages and drug effects. There is no general equation that fits all of the dose-response curves and hence the need exists to evaluate results over a range of effects (50-90\% inhibition) (Martinez-Irujo et al., 1996). We evaluated drug combinations with TLF in a systematic manner at three different dose effects $\left(\mathrm{ED}_{50}, \mathrm{ED}_{75}\right.$ and $\mathrm{ED}_{90}$ ) and multiple drug-dose ratios that may be relevant clinically. We confirmed that our data fitted the median effects equation (mean $r$ value $\geqslant 0.95$ ), rendering calculation of the CIs and DRIs valid (Chou, 2006).

Cls for drug combinations with TLF. In the eight strains of CoNS tested, the TLF and NAF combination was synergistic $(\mathrm{CI}<1)$ over a wide range of drug-dose ratios at dose effects of 50, 75 and $90 \%$ with very few exceptions (all exceptions were at $\mathrm{ED}_{50}$ : S. epidermidis ATCC 55133 at a ratio of $8000: 1$ and CoNS 103A at $64000: 1$ and $32000: 1$; Table 2). Similarly, the TLF and VAN combination was synergistic $(\mathrm{CI}<1)$ at multiple drug-dose ratios at $\mathrm{ED}_{50}$, $\mathrm{ED}_{75}$ and $\mathrm{ED}_{90}$ with no exceptions.

In the three strains of $C$. albicans tested, the TLF and AMB combination was synergistic $(\mathrm{CI}<1)$ over a wide range of drug-dose ratios at $\mathrm{ED}_{50}, \mathrm{ED}_{75}$ and $\mathrm{ED}_{90}$ with no exceptions (Table 3). Similarly, the TLF and FLC combination was synergistic $(\mathrm{CI}<1)$ at multiple drugdose ratios at $\mathrm{ED}_{50}, \mathrm{ED}_{75}$ and $\mathrm{ED}_{90}$ with only one exception $\left(\mathrm{ED}_{50}\right.$ : C. albicans ATCC 32354 at a ratio of $\left.8000: 1\right)$.
Thus TLF acted synergistically with NAF and VAN against CoNS including S. epidermidis, and with AMB and FLC against C. albicans, at most drug-dose ratios with very few exceptions. These exceptions may be explained by the inherent variability of the chequerboard method due to variability in the assay conditions on different days.

The synergy of lactoferrin with antibiotics commonly used against isolates of organisms of clinical interest is of great clinical relevance, as it has the potential for more effective therapy against drug-resistant strains and for a reduction in drug dosage. Leitch \& Willcox (1999b) reported that $1024 \mu \mathrm{g}$ lactoferrin $\mathrm{ml}^{-1}$ reduced the minimum bactericidal concentrations of VAN required to kill S. epidermidis twofold. Kuipers et al. (1999) reported significant cooperative activity of lactoferrin and apolactoferrin (ironfree) with FLC, AMB and 5-fluorocytosine against clinical Candida isolates. Peptides derived from human lactoferrin containing the first $11 \mathrm{~N}$-terminal residues were effective in making fluconazole-resistant C. albicans sensitive to fluconazole (Leitch \& Willcox, 1999a; Wakabayashi et al., 1996, 1998).

The mechanism of the synergistic effect of lactoferrin with antifungal and anti-staphylococcal agents has not been fully validated. It appears that the synergistic effect is not due to the iron-sequestering effects of lactoferrin, as both apolactoferrin and iron-saturated lactoferrin have similar activities in vitro (Kuipers et al., 1999). A direct effect on the cell membranes of Candida and staphylococci is more likely (Leitch \& Willcox, 1999a; Xu et al., 1999). We speculate that the action of lactoferrin on cell membranes complements the action of other drugs and may be responsible for the synergy.

DRIs for drug combinations with TLF. For CoNS, the DRIs indicated a significant reduction in the doses of NAF and VAN required when used in combination with TLF for a given effect, but varied widely among the strains of CoNS tested and also among the different drug-dose ratios tested in a single strain (Table 4). The DRI ranges for NAF in combination with TLF were: $2.5-10.4$ against $S$. epidermidis ATCC 55133; 5.3-14.3 against S. epidermidis $100 \mathrm{H} ; 2.1-6.6$ against S. epidermidis 101S; 11.7-176 against CoNS 102R; 1.7-7.3 against CoNS 103A; $1.7-4.3$ against CoNS 104O; 3.7-90.3 against CoNS 105D; and 1.5-4.2 against CoNS 106F. The DRI ranges for VAN in combination with TLF were: 1.6-5.9 against $S$. epidermidis ATCC 55133; 2.2-6.4 against $S$. epidermidis $100 \mathrm{H} ; 2.3-4.8$ against $S$. epidermidis 101S; 2.7-9.2 against CoNS 102R; 1.2-3.2 against CoNS 103A; 1.6-4.1 against CoNS 104O; 1.8-4.2 against CoNS 105D; and 1.7-4.9 against CoNS 106F.

For C. albicans, the DRIs indicated a significant reduction in the doses of AMB and FLC required when used in combination with TLF for a given effect, but varied widely among the strains of $C$. albicans tested and also among the different drug-dose ratios tested in a single strain (Table 5). The DRI ranges for AMB in combination with TLF were: 
Table 2. Cls for drug combinations with TLF against CoNS

CIs were derived using the median effects principle. The effect was synergistic for $\mathrm{CI}<1$, additive for $\mathrm{CI}=1$ and antagonistic for $\mathrm{CI}>1$. Results are shown as means (SD). TLF was synergistic across most drug-dose ratios (exceptions indicated in bold) and for different dose effects against clinical isolates of CoNS. ND, Not done.

\begin{tabular}{|c|c|c|c|c|c|c|c|c|}
\hline & \multirow{2}{*}{$\begin{array}{l}\text { TLF : NAF } \\
\quad \text { ratio }\end{array}$} & \multicolumn{3}{|c|}{ CI for TLF with NAF } & \multirow[t]{2}{*}{ TLF : VAN ratio } & \multicolumn{3}{|c|}{ CI for TLF with VAN } \\
\hline & & $\mathrm{ED}_{50}$ & $\mathrm{ED}_{75}$ & $\mathrm{ED}_{90}$ & & $\mathrm{ED}_{50}$ & $\mathrm{ED}_{75}$ & $\mathrm{ED}_{90}$ \\
\hline \multirow{4}{*}{$\begin{array}{l}\text { S. epidermidis } \\
\text { ATCC } 55133\end{array}$} & $16000: 1$ & $0.25(0.04)$ & $0.18(0.04)$ & $0.14(0.03)$ & $2000: 1$ & $0.54(0.25)$ & $0.36(0.02)$ & $0.31(0.08)$ \\
\hline & $8000: 1$ & $1.04(1.16)$ & $0.44(0.36)$ & $0.21(0.07)$ & $1000: 1$ & $0.49(0.13)$ & $0.40(0.01)$ & $0.38(0.09)$ \\
\hline & $2000: 1$ & $0.73(0.65)$ & $0.44(0.28)$ & $0.28(0.08)$ & $250: 1$ & $0.48(0.16)$ & $0.33(0.13)$ & $0.24(0.12)$ \\
\hline & $1000: 1$ & $0.33(0.19)$ & $0.37(0.17)$ & $0.42(0.13)$ & $125: 1$ & $0.65(0.15)$ & $0.55(0.03)$ & $0.50(0.16)$ \\
\hline \multirow{3}{*}{$\begin{array}{l}\text { S. epidermidis } \\
100 \mathrm{H}\end{array}$} & $8000: 1$ & $0.41(0.01)$ & $0.22(0.07)$ & $0.17(0.05)$ & $2000: 1$ & $0.39(0.00)$ & $0.33(0.03)$ & $0.38(0.05)$ \\
\hline & $4000: 1^{\star}$ & $0.43(0.01)$ & $0.24(0.06)$ & $0.15(0.07)$ & $1000: 1$ & $0.41(0.08)$ & $0.35(0.10)$ & $0.37(0.06)$ \\
\hline & $2000: 1$ & $0.40(0.09)$ & $0.25(0.10)$ & $0.18(0.08)$ & $500: 1^{\star}$ & $0.44(0.15)$ & $0.40(0.16)$ & $0.40(0.13)$ \\
\hline \multirow{4}{*}{$\begin{array}{l}\text { S. epidermidis } \\
101 \mathrm{~S}\end{array}$} & $8000: 1^{\star}$ & $0.43(0.24)$ & $0.29(0.09)$ & $0.22(0.03)$ & $1000: 1$ & $0.36(0.11)$ & $0.29(0.08)$ & $0.27(0.09)$ \\
\hline & $4000: 1$ & $0.32(0.01)$ & $0.29(0.0)$ & $0.27(0.0)$ & $500: 1$ & $0.39(0.10)$ & $0.32(0.09)$ & $0.28(0.10)$ \\
\hline & $2000: 1$ & $0.41(0.02)$ & $0.35(0.01)$ & $0.31(0.01)$ & $250: 1^{*}$ & $0.43(0.09)$ & $0.36(0.08)$ & $0.32(0.08)$ \\
\hline & $1000: 1$ & $0.50(0.05)$ & $0.42(0.02)$ & $0.36(0.0)$ & $125: 1$ & $0.48(0.13)$ & $0.41(0.09)$ & $0.36(0.08)$ \\
\hline \multirow[t]{5}{*}{ CoNS 102R } & $16000: 1^{\star}$ & $0.13(0.16)$ & $0.17(0.02)$ & $0.66(0.71)$ & $2000: 1$ & $0.44(0.15)$ & $0.38(0.04)$ & $0.36(0.18)$ \\
\hline & $8000: 1$ & $0.12(0.10)$ & $0.15(0.02)$ & $0.26(0.17)$ & $1000: 1$ & $0.38(0.11)$ & $0.32(0.00)$ & $0.28(0.06)$ \\
\hline & $4000: 1$ & $0.11(0.09)$ & $0.11(0.04)$ & $0.13(0.01)$ & $500: 1^{\star}$ & $0.38(0.08)$ & $0.32(0.02)$ & $0.28(0.02)$ \\
\hline & $2000: 1$ & $0.12(0.09)$ & $0.12(0.04)$ & $0.14(0.00)$ & $250: 1$ & $0.41(0.02)$ & $0.34(0.01)$ & $0.30(0.01)$ \\
\hline & $1000: 1$ & $0.13(0.09)$ & $0.12(0.04)$ & $0.13(0.01)$ & $125: 1$ & $0.44(0.07)$ & $0.40(0.03)$ & $0.37(0.01)$ \\
\hline \multirow[t]{2}{*}{ CoNS 103A } & $64000: 1^{\star}$ & $1.24(0.32)$ & $0.33(0.03)$ & $0.17(0.03)$ & $2000: 1^{\star}$ & $0.63(0.26)$ & $0.37(0.03)$ & $0.32(0.01)$ \\
\hline & $32000: 1$ & $1.05(0.32)$ & $0.33(0.02)$ & $0.18(0.04)$ & $1000: 1$ & $0.93(0.68)$ & $0.47(0.19)$ & $0.33(0.07)$ \\
\hline \multirow{3}{*}{ CoNS $104 \mathrm{O}$} & $4000: 1$ & $0.52(0.05)$ & $0.41(0.03)$ & $0.37(0.13)$ & $500: 1^{\star}$ & $0.43(0.04)$ & $0.53(0.20)$ & $0.70(0.44)$ \\
\hline & $2000: 1$ & $0.53(0.04)$ & $0.47(0.02)$ & $0.44(0.09)$ & $250: 1$ & $0.50(0.03)$ & $0.55(0.19)$ & $0.65(0.39)$ \\
\hline & $1000: 1$ & $0.62(0.04)$ & $0.56(0.03)$ & $0.52(0.09)$ & $125: 1$ & $0.56(0.01)$ & $0.64(0.20)$ & $0.77(0.44)$ \\
\hline \multirow[t]{5}{*}{ CoNS 105D } & $16000: 1$ & $0.55(0.17)$ & $0.24(0.1)$ & $0.12(0.08)$ & $2000: 1$ & $0.47(0.12)$ & $0.35(0.10)$ & $0.37(0.24)$ \\
\hline & $8000: 1^{\star}$ & $0.45(0.20)$ & $0.22(0.10)$ & $0.12(0.07)$ & $1000: 1$ & $0.44(0.06)$ & $0.35(0.14)$ & $0.36(0.26)$ \\
\hline & $4000: 1$ & $0.40(0.23)$ & $0.21(0.11)$ & $0.12(0.07)$ & $500: 1^{\star}$ & $0.44(0.02)$ & $0.40(0.14)$ & $0.43(0.28)$ \\
\hline & $2000: 1$ & $0.40(0.18)$ & $0.25(0.10)$ & $0.17(0.06)$ & $250: 1$ & $0.49(0.05)$ & $0.45(0.15)$ & $0.45(0.23)$ \\
\hline & $1000: 1$ & $0.45(0.21)$ & $0.31(0.12)$ & $0.22(0.08)$ & $125: 1$ & $0.59(0.03)$ & $0.53(0.18)$ & $0.50(0.30)$ \\
\hline \multirow[t]{6}{*}{ CoNS 106F } & $32000: 1^{\star}$ & $0.98(0.48)$ & $0.37(0.07)$ & $0.25(0.01)$ & $2000: 1$ & $0.42(0.03)$ & $0.31(0.02)$ & $0.26(0.06)$ \\
\hline & $16000: 1$ & $0.74(0.18)$ & $0.35(0.05)$ & $0.25(0.05)$ & $1000: 1$ & $0.44(0.01)$ & $0.33(0.03)$ & $0.28(0.04)$ \\
\hline & $8000: 1$ & $0.66(0.07)$ & $0.40(0.02)$ & $0.31(0.04)$ & $500: 1^{\star}$ & $0.45(0.0)$ & $0.36(0.02)$ & $0.31(0.04)$ \\
\hline & $4000: 1$ & $0.45(0.02)$ & $0.32(0.14)$ & $0.26(0.09)$ & $250: 1$ & $0.49(0.01)$ & $0.41(0.04)$ & $0.35(0.07)$ \\
\hline & $2000: 1$ & $0.66(0.04)$ & $0.47(0.01)$ & $0.36(0.01)$ & $125: 1$ & $0.61(0.05)$ & $0.49(0.02)$ & $0.40(0.07)$ \\
\hline & $1000: 1$ & $0.71(0.03)$ & $0.51(0.05)$ & $0.39(0.06)$ & $62.5: 1$ & $0.69(0.32)$ & $0.46(0.22)$ & $0.30(0.15)$ \\
\hline
\end{tabular}

${ }^{\star}$ Represents equipotency ratios (ratios of $\mathrm{MIC}_{50}$ ). 
Table 3. Cls for drug combinations with TLF against C. albicans

CIs were derived by the median effects principle. The effect was synergistic for $\mathrm{CI}<1$, additive for CI $=1$ and antagonistic for CI $>1$. Results are shown as means (SD). TLF was synergistic across most drug-dose ratios (exception indicated in bold) and for different dose effects against clinical isolates of C. albicans. ND, Not done.

\begin{tabular}{|c|c|c|c|c|c|c|c|c|}
\hline \multirow[t]{2}{*}{ Organism } & \multirow[t]{2}{*}{ TLF : AMB ratio } & \multicolumn{3}{|c|}{ CI for TLF with AMB } & \multirow[t]{2}{*}{ TLF : FLC ratio } & \multicolumn{3}{|c|}{ CI for TLF with FLC } \\
\hline & & $\mathrm{ED}_{50}$ & $\mathrm{ED}_{75}$ & $\mathrm{ED}_{90}$ & & $\mathrm{ED}_{50}$ & $\mathbf{E D}_{75}$ & $\mathrm{ED}_{90}$ \\
\hline \multirow{5}{*}{$\begin{array}{l}\text { C. albicans ATCC } \\
32354\end{array}$} & $4000: 1$ & $0.08(0.08)$ & $0.07(0.07)$ & $0.08(0.05)$ & $4000: 1$ & $0.79(0.64)$ & $0.21(0.01)$ & $0.09(0.08)$ \\
\hline & $2000: 1^{\star}$ & $0.11(0.08)$ & $0.11(0.08)$ & $0.12(0.05)$ & $2000: 1^{\star}$ & $0.47(0.21)$ & $0.19(0.09)$ & $0.11(0.12)$ \\
\hline & $250: 1$ & $0.21(0.29)$ & $0.16(0.23)$ & $0.13(0.17)$ & $250: 1$ & $0.32(0.07)$ & $0.22(0.15)$ & $0.17(0.17)$ \\
\hline & $125: 1$ & $0.27(0.38)$ & $0.20(0.28)$ & $0.15(0.21)$ & ND & ND & ND & ND \\
\hline & $62.5: 1$ & $0.57(0.80)$ & $0.33(0.46)$ & $0.20(0.25)$ & $\mathrm{ND}$ & $\mathrm{ND}$ & $\mathrm{ND}$ & $\mathrm{ND}$ \\
\hline \multirow[t]{2}{*}{ C. albicans 200C } & $8000: 1$ & $0.22(0.09)$ & $0.14(0.03)$ & $0.09(0.01)$ & $8000: 1$ & $0.13(0.03)$ & $0.06(0.03)$ & $0.03(0.03)$ \\
\hline & $4000: 1^{\star}$ & $0.23(0.12)$ & $0.15(0.03)$ & $0.11(0.03)$ & $4000: 1$ & $0.11(0.03)$ & $0.06(0.03)$ & $0.03(0.03)$ \\
\hline \multirow[t]{6}{*}{ C. albicans $201 \mathrm{M}$} & $8000: 1$ & $0.41(0.02)$ & $0.24(0.01)$ & $0.18(0.00)$ & $8000: 1$ & $0.35(0.21)$ & $0.06(0.01)$ & $0.02(0.01)$ \\
\hline & $4000: 1^{*}$ & $0.38(0.06)$ & $0.26(0.01)$ & $0.21(0.02)$ & $4000: 1$ & $0.18(0.04)$ & $0.05(0.02)$ & $0.02(0.01)$ \\
\hline & $2000: 1$ & $0.40(0.04)$ & $0.30(0.01)$ & $0.23(0.00)$ & $2000: 1$ & $0.15(0.03)$ & $0.05(0.03)$ & $0.02(0.01)$ \\
\hline & $1000: 1$ & $0.46(0.05)$ & $0.35(0.02)$ & $0.27(0.01)$ & $1000: 1^{\star}$ & $0.15(0.07)$ & $0.06(0.04)$ & $0.03(0.02)$ \\
\hline & $500: 1$ & $0.73(0.17)$ & $0.49(0.09)$ & $0.34(0.05)$ & $500: 1$ & $0.23(0.13)$ & $0.09(0.06)$ & $0.04(0.03)$ \\
\hline & $250: 1$ & $0.35(0.21)$ & $0.31(0.15)$ & $0.29(0.10)$ & $250: 1$ & $0.32(0.24)$ & $0.13(0.10)$ & $0.05(0.04)$ \\
\hline
\end{tabular}

${ }^{\star}$ Represents equipotency ratios (ratios of $\mathrm{MIC}_{50}$ ).

8.2-2960 against C. albicans ATCC 32354; 4.3-12.7 for C. albicans 200C; and 1.5-5.6 for C. albicans 201M. The DRIs for FLC in combination with TLF were: 4.1-820 against $C$. albicans ATCC 32354; 8.8-177.5 against C. albicans 200C; and 4.9-77.5 against C. albicans $201 \mathrm{M}$.

We chose to report DRIs, which estimate the degree by which antibiotic dosage can be reduced in a synergistic drug combination at a given inhibitory effect. The DRIs were significant but variable among the different strains of organism tested and within the same strain at different drug-dose ratios and drug effects. The DRIs indicated twofold to several hundred-fold reductions in drug doses required and may indicate an advantage of using TLF in conjunction with antibiotics in the treatment or prevention of neonatal sepsis. DRIs are not commonly reported in the literature, but the clinical relevance underlines their importance.

\section{Limitations of the study}

The limitations of this study follow the inherent variability of chequerboard assays and hence the need for repeated assays. We repeated our experiments in duplicate and on two different days, and the readings on any particular day were carried out under the same experimental conditions and were averaged, generating two sets of data for analysis. Chequerboard combinations used serial twofold dilutions of the drug, but the effects of the drug concentrations between the twofold dilutions were not evaluated, which may be valuable in future studies. We did not test concentrations higher than $8300 \mu \mathrm{g} \mathrm{ml} \mathrm{m}^{-1}$ for TLF, although some studies using higher concentrations (Kuipers et al., 1999) have shown complete inhibition of Candida isolates. The general limitation of experiments in vitro in terms of correlation with effects in vivo that exist may be due to the physical environment as well as the participation of other host defence mechanisms. Antimicrobial combinations need to be evaluated in appropriate animal models using clinical isolates from organisms of interest.

\section{Conclusions}

The antimicrobial activity of lactoferrins in general, and TLF in particular, against common neonatal isolates of CoNS (including S. epidermidis) and C. albicans, and the synergistic effect with antibiotics commonly used in neonatal practice, make TLF a promising agent in the 
Table 4. DRls for drug combinations with TLF against CoNS

DRIs were determined using the median effects principle. Results are shown as means (SD). The DRIs suggest that a significant reduction in antibiotic dosage is possible when combined with TLF across drug-dose ratios and for different dose effects against CoNS. ND, Not done.

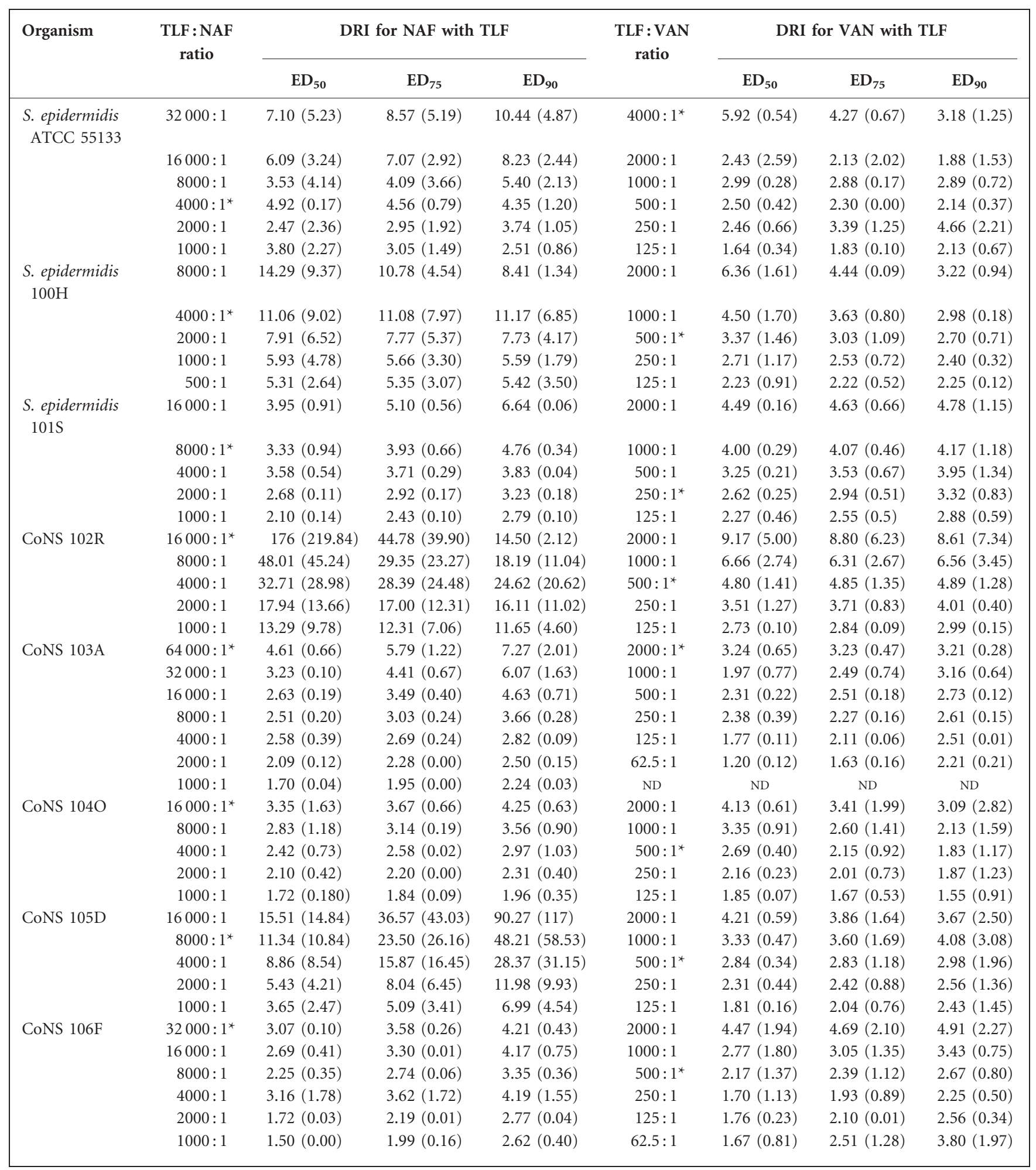

${ }^{\star}$ Represents equipotency ratios (ratios of $\mathrm{MIC}_{50}$ ). 
Table 5. DRls for drug combinations with TLF against C. albicans

DRIs were determined using the median effects principle. Results are shown as means (SD). The DRIs suggest that a significant reduction in antibiotic dosages is possible when combined with TLF across various drug-dose ratios and for different dose effects against clinical isolates of $C$. albicans.

\begin{tabular}{|c|c|c|c|c|c|c|c|c|}
\hline Organism & $\begin{array}{l}\text { TLF : AMB } \\
\quad \text { ratio }\end{array}$ & $\mathrm{ED}_{50}$ & $\mathrm{ED}_{75}$ & $\mathrm{ED}_{90}$ & $\begin{array}{l}\text { TLF : FLC } \\
\quad \text { ratio }\end{array}$ & $\mathrm{ED}_{50}$ & $\mathrm{ED}_{75}$ & $\mathrm{ED}_{90}$ \\
\hline \multirow{5}{*}{$\begin{array}{l}\text { C. albicans } \\
\text { ATCC } 32354\end{array}$} & $4000: 1$ & $82.5(105.20)$ & $36.63(39.70)$ & $17.82(12.42)$ & $4000: 1$ & $70.41(92.8)$ & $213.80(293)$ & $819.5(1146)$ \\
\hline & $500: 1$ & $58.90(75.10)$ & $26.22(29.25)$ & $12.44(10.12)$ & $500: 1$ & $4.18(2.16)$ & $9.56(8.55)$ & $23.62(26.80)$ \\
\hline & $250: 1$ & $2552(3605)$ & $289(404)$ & $34.38(43.03)$ & $250: 1$ & $4.10(1.84)$ & $7.26(5.85)$ & $13.85(14.60)$ \\
\hline & $125: 1$ & $2960(4184)$ & $1632(2304)$ & $91.57(124.8)$ & ND & ND & ND & $\mathrm{ND}$ \\
\hline & $62.5: 1$ & $1134(1603)$ & $156(218)$ & $22.67(28.33)$ & $\mathrm{ND}$ & $\mathrm{ND}$ & ND & $\mathrm{ND}$ \\
\hline \multirow[t]{3}{*}{ C. albicans $200 \mathrm{C}$} & $8000: 1$ & $6.39(3.83)$ & $8.21(1.97)$ & $11.40(1.7)$ & $8000: 1$ & $17.19(9.35)$ & $53.65(46.32)$ & $177.54(192)$ \\
\hline & $4000: 1^{\star}$ & $5.88(3.71)$ & $7.09(1.43)$ & $9.52(2.58)$ & $4000: 1$ & $14.98(7.10)$ & $41.89(33.82)$ & 123.73 (129) \\
\hline & $250: 1$ & $5.40(2.69)$ & $5.81(3.55)$ & $6.33(4.43)$ & $250: 1$ & $8.79(6.10)$ & $20.67(18.01)$ & $49.53(50.20)$ \\
\hline \multirow[t]{6}{*}{ C. albicans $201 \mathrm{M}$} & $8000: 1$ & $3.23(0.10)$ & $4.27(0.05)$ & $5.63(0.05)$ & $8000: 1$ & $11.00(6.08)$ & $29.18(17.37)$ & $77.46(49.30)$ \\
\hline & $4000: 1^{\star}$ & $3.14(0.48)$ & $3.89(0.13)$ & $4.88(0.45)$ & $4000: 1$ & $14.37(9.01)$ & $29.62(18.84)$ & $61.14(39.20)$ \\
\hline & $2000: 1$ & $2.71(0.30)$ & $3.43(0.18)$ & $4.36(0.06)$ & $2000: 1$ & $13.04(9.67)$ & $26.39(17.80)$ & $53.50(32.40)$ \\
\hline & $1000: 1$ & $2.26(0.23)$ & $2.91(0.16)$ & $3.74(0.06)$ & $1000: 1^{\star}$ & $11.28(8.60)$ & $21.87(15.65)$ & $42.35(28.50)$ \\
\hline & $500: 1$ & $1.45(0.35)$ & $2.04(0.36)$ & $2.98(0.45)$ & $500: 1$ & $6.74(5.01)$ & $16.02(11.91)$ & $37.98(28.40)$ \\
\hline & $250: 1$ & $3.53(2.16)$ & $3.59(1.68)$ & $3.66(1.22)$ & $250: 1$ & $4.89(3.94)$ & $11.73(9.51)$ & $28.39(22.60)$ \\
\hline
\end{tabular}

${ }^{\star}$ Represents equipotency ratios (ratios of $\mathrm{MIC}_{50}$ ).

therapy and prevention of neonatal sepsis. Lactoferrin has been effective in animal models of infection and in polymicrobial infections in vivo. Clinical studies are needed to evaluate the potential efficacy of TLF in the treatment and prophylaxis of neonates against systemic infections to reduce mortality and morbidity.

\section{ACKNOWLEDGEMENTS}

We acknowledge the help of Agennix for donating TLF for our research. We also thank C. Fernandes and Bhagvatula Moorthy for critically reviewing the manuscript.

\section{REFERENCES}

Adams-Chapman, I. \& Stoll, B. J. (2006). Neonatal infection and long-term neurodevelopmental outcome in the preterm infant. Curr Opin Infect Dis 19, 290-297.

Baker, E. N., Anderson, B. F., Baker, H. M., MacGillivray, R. T., Moore, S. A., Peterson, N. A., Shewry, S. C. \& Tweedie, J. W. (1998). Threedimensional structure of lactoferrin. Implications for function, including comparisons with transferrin. Adv Exp Med Biol 443, 1-14.

Bennett, R. M. \& Kokocinski, T. (1978). Lactoferrin content of peripheral blood cells. Br J Haematol 39, 509-521.
Berenbaum, M. C. (1989). What is synergy? Pharmacol Rev 41, 93141.

Cerca, N., Martins, S., Cerca, F., Jefferson, K. K., Pier, G. B., Oliveira, R. \& Azeredo, J. (2005). Comparative assessment of antibiotic susceptibility of coagulase-negative staphylococci in biofilm versus planktonic culture as assessed by bacterial enumeration or rapid XTT colorimetry. J Antimicrob Chemother 56, 331-336.

Chou, T. C. (2006). Theoretical basis, experimental design, and computerized simulation of synergism and antagonism in drug combination studies. Pharmacol Rev 58, 621-681.

CLSI (2002). Reference Method for Broth Dilution Antifungal Susceptibility Testing of Yeasts, 2nd edn. Approved Standard M27A2. Wayne, PA: Clinical and Laboratory Standards Institute.

CLSI (2003). Methods for Dilution Antimicrobial Susceptibility Tests for Bacteria that Grow Aerobically, 6th edn. Approved Standard M7-A6. Wayne, PA: Clinical and Laboratory Standards Institute.

CLSI (2007). Performance Standards for Antimicrobial Susceptibility Testing, 17th Informational Supplement, M100-S17. Wayne, PA: Clinical and Laboratory Standards Institute.

Edde, L., Hipolito, R. B., Hwang, F. F., Headon, D. R., Shalwitz, R. A. \& Sherman, M. P. (2001). Lactoferrin protects neonatal rats from gutrelated systemic infection. Am J Physiol Gastrointest Liver Physiol 281, G1140-G1150.

Farnaud, S. \& Evans, R. W. (2003). Lactoferrin - a multifunctional protein with antimicrobial properties. Mol Immunol 40, 395-405. 
Greco, W. R., Bravo, G. \& Parsons, J. C. (1995). The search for synergy: a critical review from a response surface perspective. Pharmacol Rev 47, 331-385.

Hennart, P. F., Brasseur, D. J., Delogne-Desnoeck, J. B., Dramaix, M. M. \& Robyn, C. E. (1991). Lysozyme, lactoferrin, and secretory immunoglobulin A content in breast milk: influence of duration of lactation, nutrition status, prolactin status, and parity of mother. Am J Clin Nutr 53, 32-39.

Kalmar, J. R. \& Arnold, R. R. (1988). Killing of Actinobacillus actinomycetemcomitans by human lactoferrin. Infect Immun 56, 2552 2557.

Kaufman, D. \& Fairchild, K. D. (2004). Clinical microbiology of bacterial and fungal sepsis in very-low-birth-weight infants. Clin Microbiol Rev 17, 638-680.

Kuipers, M. E., de Vries, H. G., Eikelboom, M. C., Meijer, D. K. \& Swart, P. J. (1999). Synergistic fungistatic effects of lactoferrin in combination with antifungal drugs against clinical Candida isolates. Antimicrob Agents Chemother 43, 2635-2641.

Lawn, J. E., Wilczynska-Ketende, K. \& Cousens, S. N. (2006). Estimating the causes of 4 million neonatal deaths in the year 2000. Int J Epidemiol 35, 706-718.

Leitch, E. C. \& Willcox, M. D. (1999a). Elucidation of the antistaphylococcal action of lactoferrin and lysozyme. $J \mathrm{Med}$ Microbiol 48, 867-871.

Leitch, E. C. \& Willcox, M. D. (1999b). Lactoferrin increases the susceptibility of $S$. epidermidis biofilms to lysozyme and vancomycin. Curr Eye Res 19, 12-19.

Levy, S. B. (1998). The challenge of antibiotic resistance. Sci Am 278, 46-53.

Levy, S. B. (2001). Antimicrobial resistance potential. Lancet 358, $1100-1101$.

Lupetti, A., Paulusma-Annema, A., Welling, M. M., Senesi, S., van Dissel, J. T. \& Nibbering, P. H. (2000). Candidacidal activities of human lactoferrin peptides derived from the $\mathrm{N}$ terminus. Antimicrob Agents Chemother 44, 3257-3263.

Lupetti, A., Paulusma-Annema, A., Welling, M. M., DogteromBallering, H., Brouwer, C. P., Senesi, S., Van Dissel, J. T. \& Nibbering, P. H. (2003). Synergistic activity of the N-terminal peptide of human lactoferrin and fluconazole against Candida species. Antimicrob Agents Chemother 47, 262-267.

Lupetti, A., Brouwer, C. P., Bogaards, S. J., Welling, M. M., de Heer, E., Campa, M., van Dissel, J. T., Friesen, R. H. \& Nibbering, P. H. (2007). Human lactoferrin-derived peptide's antifungal activities against disseminated Candida albicans infection. J Infect Dis 196, 1416-1424.

Martinez-Irujo, J. J., Villahermosa, M. L., Alberdi, E. \& Santiago, E. (1996). A checkerboard method to evaluate interactions between drugs. Biochem Pharmacol 51, 635-644.

Masson, P. L. \& Heremans, J. F. (1971). Lactoferrin in milk from different species. Comp Biochem Physiol B 39, 119-129.

Masson, P. L., Heremans, J. F. \& Dive, C. (1966). An iron-binding protein common to many external secretions. Clin Chim Acta 14, 735-739.
Masson, P. L., Heremans, J. F. \& Schonne, E. (1969). Lactoferrin, an iron-binding protein in neutrophilic leukocytes. J Exp Med 130, 643-658.

Moguilevsky, N., Retegui, L. A. \& Masson, P. L. (1985). Comparison of human lactoferrins from milk and neutrophilic leucocytes. Relative molecular mass, isoelectric point, iron-binding properties and uptake by the liver. Biochem J 229, 353-359.

Odds, F. C. (2003). Synergy, antagonism, and what the chequerboard puts between them. J Antimicrob Chemother 52, 1.

Orsi, N. (2004). The antimicrobial activity of lactoferrin: current status and perspectives. Biometals 17, 189-196.

Prichard, M. N., Prichard, L. E. \& Shipman, C., Jr (1993). Strategic design and three-dimensional analysis of antiviral drug combinations. Antimicrob Agents Chemother 37, 540-545.

Sanchez, L., Calvo, M. \& Brock, J. H. (1992). Biological role of lactoferrin. Arch Dis Child 67, 657-661.

Stoll, B. J., Hansen, N., Fanaroff, A. A., Wright, L. L., Carlo, W. A., Ehrenkranz, R. A., Lemons, J. A., Donovan, E. F., Stark, A. R. \& other authors (2002). Late-onset sepsis in very low birth weight neonates: the experience of the NICHD Neonatal Research Network. Pediatrics 110, 285-291.

Stoll, B. J., Hansen, N. I., Adams-Chapman, I., Fanaroff, A. A., Hintz, S. R., Vohr, B. \& Higgins, R. D. (2004). Neurodevelopmental and growth impairment among extremely low-birth-weight infants with neonatal infection. JAMA 292, 2357-2365.

Valenti, P. \& Antonini, G. (2005). Lactoferrin: an important host defence against microbial and viral attack. Cell Mol Life Sci 62, 25762587.

Venkatesh, M. P., Pham, D., Kong, L. \& Weisman, L. E. (2007). Prophylaxis with lactoferrin, a novel antimicrobial agent, in a neonatal rat model of coinfection. Adv Ther 24, 941-954.

Wakabayashi, H., Abe, S., Okutomi, T., Tansho, S., Kawase, K. \& Yamaguchi, H. (1996). Cooperative anti-Candida effects of lactoferrin or its peptides in combination with azole antifungal agents. Microbiol Immunol 40, 821-825.

Wakabayashi, H., Okutomi, T., Abe, S., Hayasawa, H., Tomita, M. \& Yamaguchi, H. (1998). Enhanced anti-Candida activity of neutrophils and azole antifungal agents in the presence of lactoferrin-related compounds. Adv Exp Med Biol 443, 229-237.

Ward, P. P., Piddington, C. S., Cunningham, G. A., Zhou, X., Wyatt, R. D. \& Conneely, O. M. (1995). A system for production of commercial quantities of human lactoferrin: a broad spectrum natural antibiotic. Biotechnology (N Y) 13, 498-503.

Ward, P. P., Paz, E. \& Conneely, O. M. (2005). Multifunctional roles of lactoferrin: a critical overview. Cell Mol Life Sci 62, 2540-2548.

Xu, Y. Y., Samaranayake, Y. H., Samaranayake, L. P. \& Nikawa, H. (1999). In vitro susceptibility of Candida species to lactoferrin. Med Mycol 37, 35-41.

Yamauchi, K., Tomita, M., Giehl, T. J. \& Ellison, R. T., III (1993). Antibacterial activity of lactoferrin and a pepsin-derived lactoferrin peptide fragment. Infect Immun 61, 719-728. 